\title{
Erratum: Electric dipole moments in the minimal scotogenic Model
}

\author{
Asmaa Abada ${ }^{a}$ and Takashi Toma ${ }^{b}$ \\ ${ }^{a}$ Laboratoire de Physique Théorique, CNRS, Univ. Paris-Sud, Université Paris-Saclay, \\ 91405 Orsay, France \\ ${ }^{b}$ Physik-Department T30d, Technische Universität München, \\ James-Franck-Straße, D-85748 Garching, Germany \\ E-mail: asmaa.abada@th.u-psud.fr, takashi.toma@tum.de
}

ERRATUM TO: JHEP04(2018)030

ArXiv EPrint: 1802.00007

The sentences below eq. (3.8) in section 3.2, figure 3-7 and appendix A are corrected. The loop function $I_{D}\left(x_{i}, x_{j}\right)$ is changed to be exactly zero in the minimal scotogenic model. In addition, the definition of $I_{1+2}^{M}\left(x_{i}, x_{j}\right)$ and $I_{3}^{M}\left(x_{i}, x_{j}\right)$ are corrected in eq. (A.1) and (A.2) in appendix since these equations were opposite in the previous published version. However the total loop function $I_{M}\left(x_{i}, x_{j}\right)=I_{1+2}^{M}\left(x_{i}, x_{j}\right)+I_{3}^{M}\left(x_{i}, x_{j}\right)$ is the same. Due to the corrections, we also changed the corresponding figures. In figure 3 , the behaviour of the loop function $I_{D}\left(x_{i}, x_{j}\right)$ does not appear as $I_{D}\left(x_{i}, x_{j}\right)$ cancels exactly. Finally, we have recomputed numerically the solutions leading to figures $4-7$ which we display, even if they lead to the same qualitative conclusions.

Below eq. (3.8) in section 3.2. The behaviour of the loop function $I_{M}\left(x_{i}, x_{j}\right)$ that has been numerically evaluated, is shown in figure 3 as a function of $x_{i}$ for several values of $x_{j}$. Note that the loop function $I_{D}\left(x_{i}, x_{j}\right)$ is exactly zero as explained in appendix. One can see that the maximal values are $\mathcal{O}(0.01)$ when there is a large hierarchy between the masses of the two sterile states, $x_{i} \ll x_{j}$ ( $i$ and $j$ can be interchanged, as discussed above). Conversely, this loop function is suppressed for larger new particle masses, $m_{i}$ and $m_{\eta^{+}}$. Furthermore, since the loop function is anti-symmetric under the exchange of $i \leftrightarrow j$, this would also be suppressed if $m_{i}$ and $m_{j}$ are extremely degenerate as can be noticed from figure 3 . 


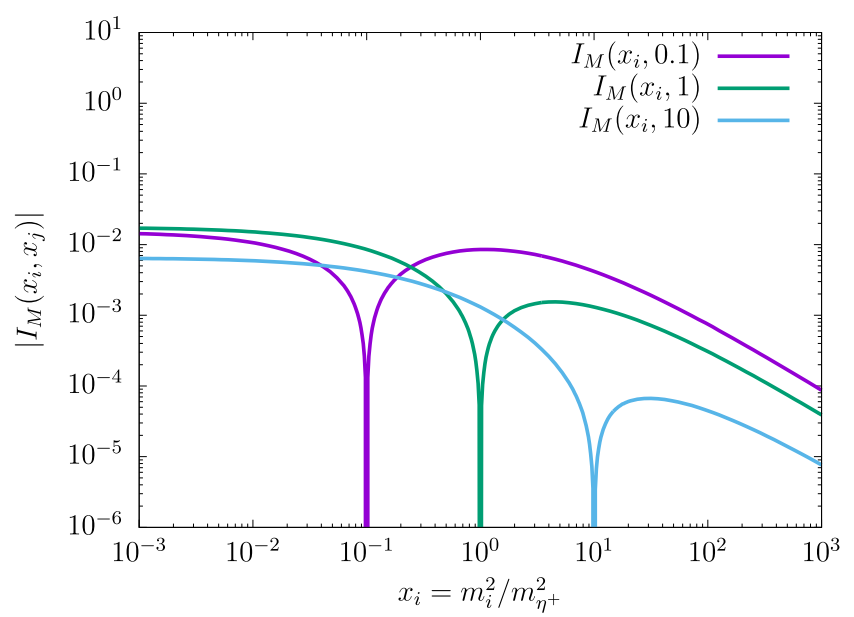

Figure 3. Loop function $I_{M}\left(x_{i}, x_{j}\right)$ as a function of $x_{i}$ where $x_{j}=m_{j}^{2} / m_{\eta^{+}}^{2}$ is fixed to $0.1,1$ and 10. The loop function $I_{D}\left(x_{i}, x_{j}\right)$ is exactly zero as explained in appendix.

Appendix A: loop functions. Here we give the loop functions which appear in the formula of charged lepton EDMs at two-loop level in eq. (3.4). The contribution from the pair of the diagrams (a1) and (a2), and the contribution from the diagram (a3) are given by

$$
\begin{aligned}
I_{1+2}^{M}\left(x_{i}, x_{j}\right)= & \int_{0}^{1} \prod_{a=1}^{3} d s_{a} \delta\left(\sum_{a=1}^{3} s_{a}-1\right) \int_{0}^{1} \prod_{b}^{4} d t_{b} \delta\left(\sum_{b=1}^{4} t_{b}-1\right) \\
& \times \frac{s_{1} s_{2}\left(1-t_{4}\right)\left(-t_{1}+s_{2} t_{1}-s_{3} t_{4}\right)}{\left[s_{2}\left(1-s_{2}\right)\left(t_{1} x_{i}+t_{2}+t_{3}\right)+t_{4}\left(s_{1} x_{j}+s_{3}\right)\right]^{2}}, \\
I_{3}^{M}\left(x_{i}, x_{j}\right)= & \int_{0}^{1} \prod_{a=1}^{4} d s_{a} \delta\left(\sum_{a=1}^{4} s_{a}-1\right) \int_{0}^{1} \prod_{b}^{4} d t_{b} \delta\left(\sum_{b=1}^{4} t_{b}-1\right) \\
& \times \frac{s_{1} s_{4}\left(1-t_{3}-t_{4}\right)^{2}-t_{1} t_{2}\left(1-s_{2}-s_{3}\right)^{2}}{2\left[\left(s_{2}+s_{3}\right)\left(1-s_{2}-s_{3}\right)\left(t_{1} x_{i}+t_{2}\right)+\left(t_{3}+t_{4}\right)\left(s_{1} x_{j}+s_{4}\right)\right]^{2}} .
\end{aligned}
$$

For the diagrams (b1) and (b2), each diagram gives non-zero contribution, however one can find that these can exactly be the same expressions with opposite sign. Thus the contributions from (b1) and (b2) cancel with each other, and the whole Majorana type loop function is given by $I_{M}\left(x_{i}, x_{j}\right)=I_{1+2}^{M}\left(x_{i}, x_{j}\right)+I_{3}^{M}\left(x_{i}, x_{j}\right)$.

The diagrams (c1) and (c2) providing the Dirac type contribution include a divergence for each diagram. However the divergence cancels out and we found that the whole loop function also exactly cancels out due to the same structure of the diagrams (b1) and (b2). Thus the Dirac type loop function exactly vanishes in the minimal scotogenic model.

Open Access. This article is distributed under the terms of the Creative Commons Attribution License (CC-BY 4.0), which permits any use, distribution and reproduction in any medium, provided the original author(s) and source are credited. 

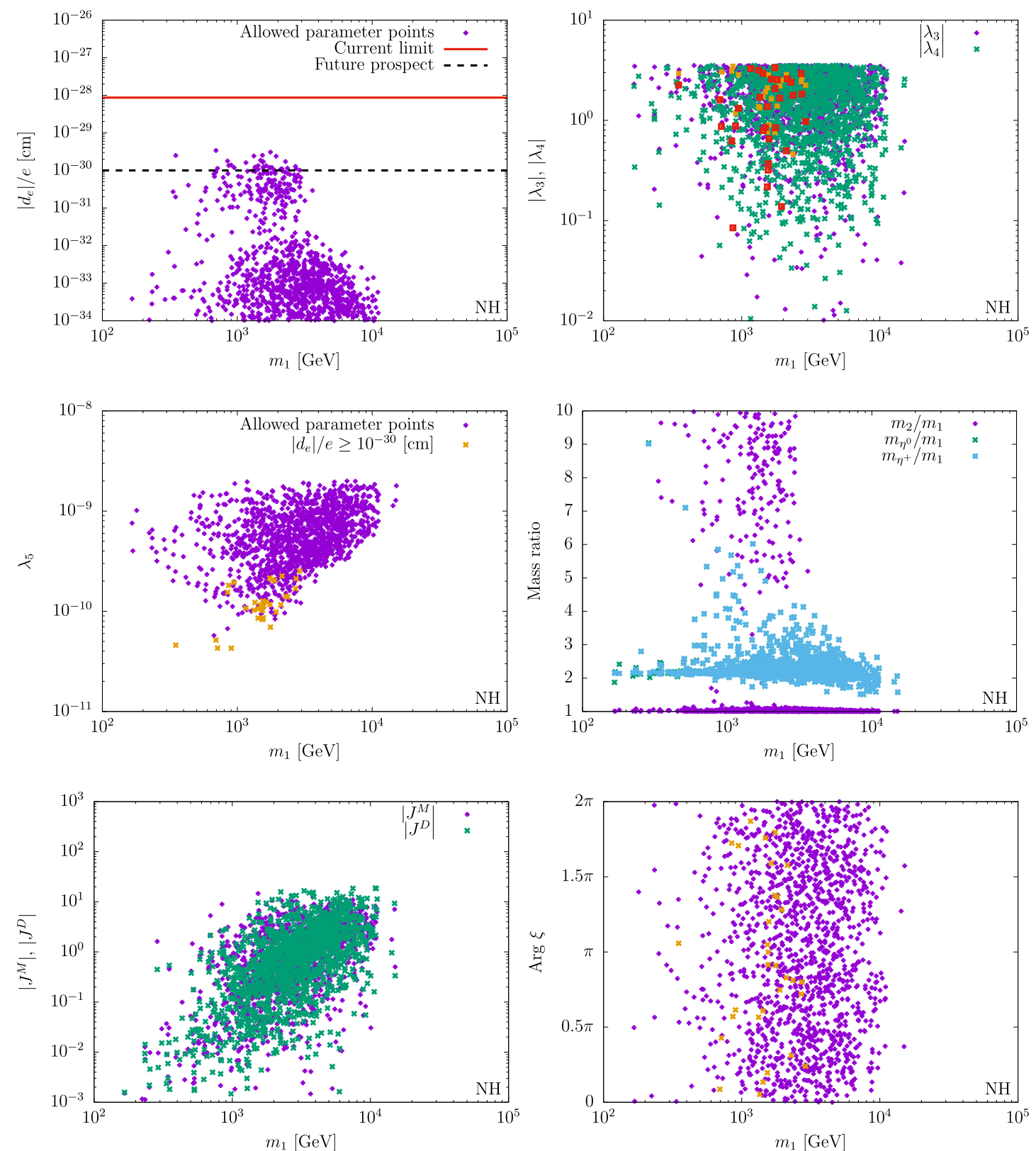

Figure 4. Numerical results for fermionic dark matter in the case of normal hierarchy for the light neutrino spectrum. Each point complies with all the constraints discussed in section 4 . 

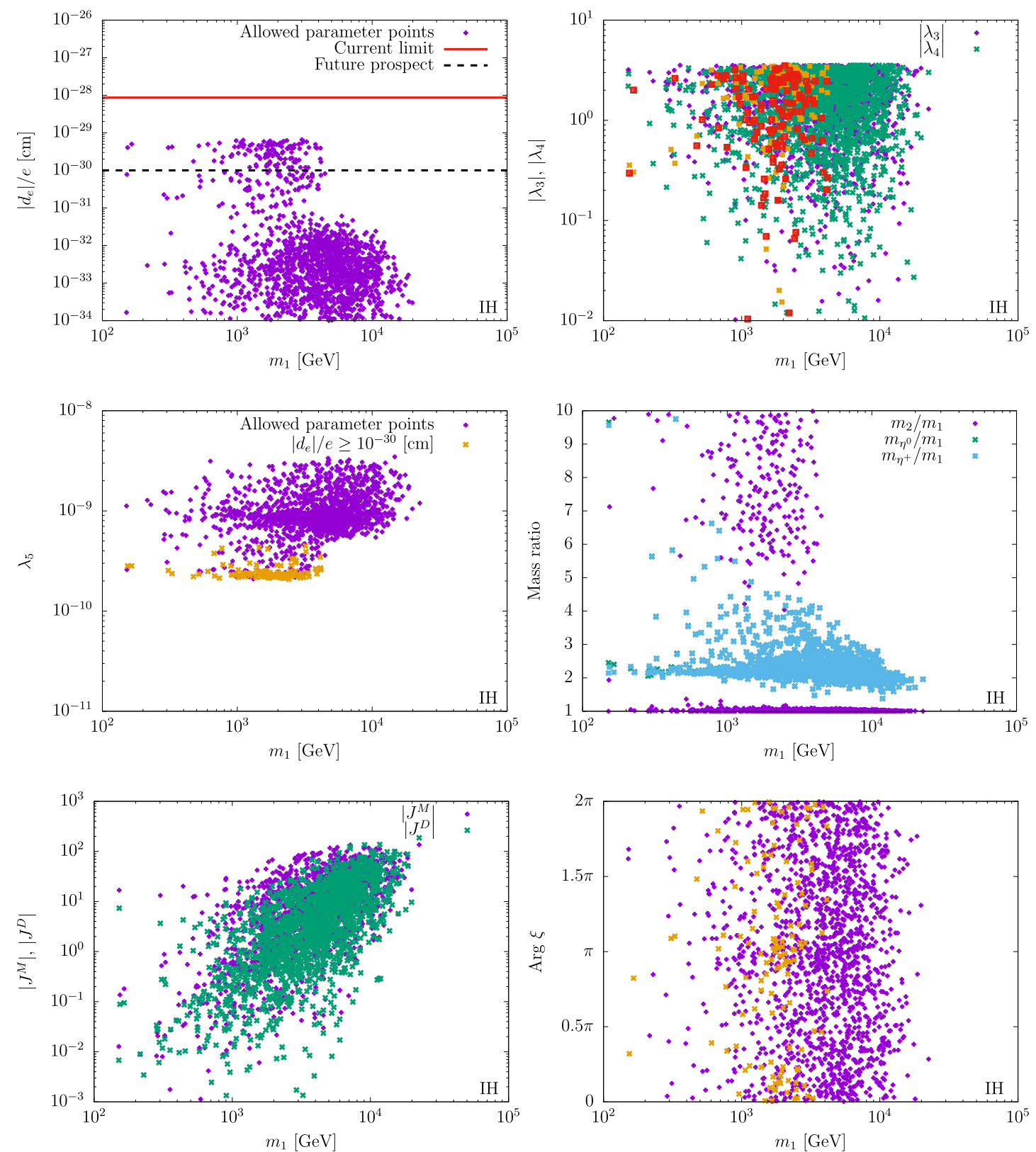

Figure 5. Numerical results for fermion dark matter in the case of inverted hierarchy for the light neutrino spectrum. Each point complies with all the constraints discussed in section 4 . 

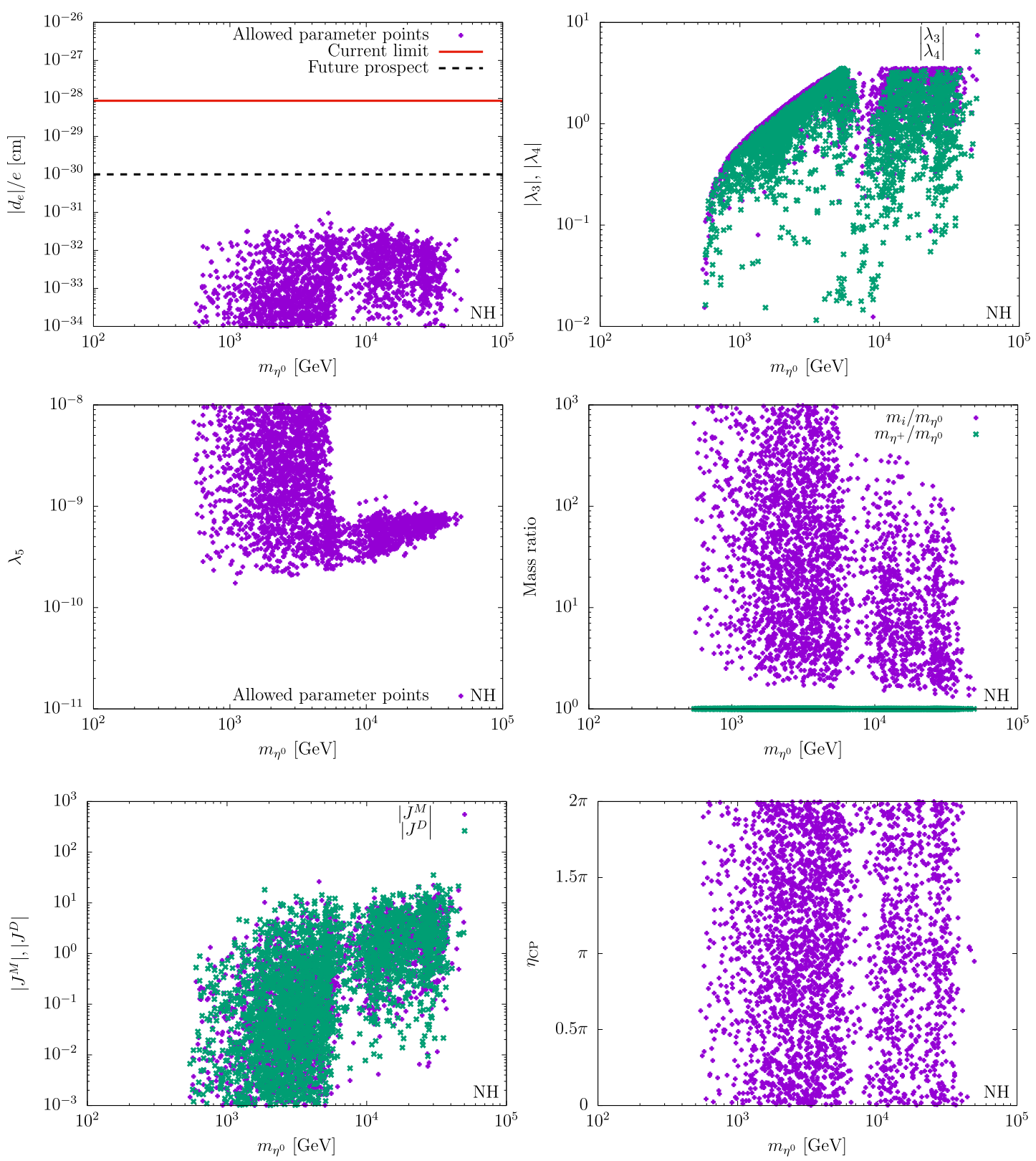

Figure 6. Numerical results for scalar dark matter in the case of normal ordering of the light neutrino mass spectrum. Each point complies with all the constraints discussed in section 4 . 

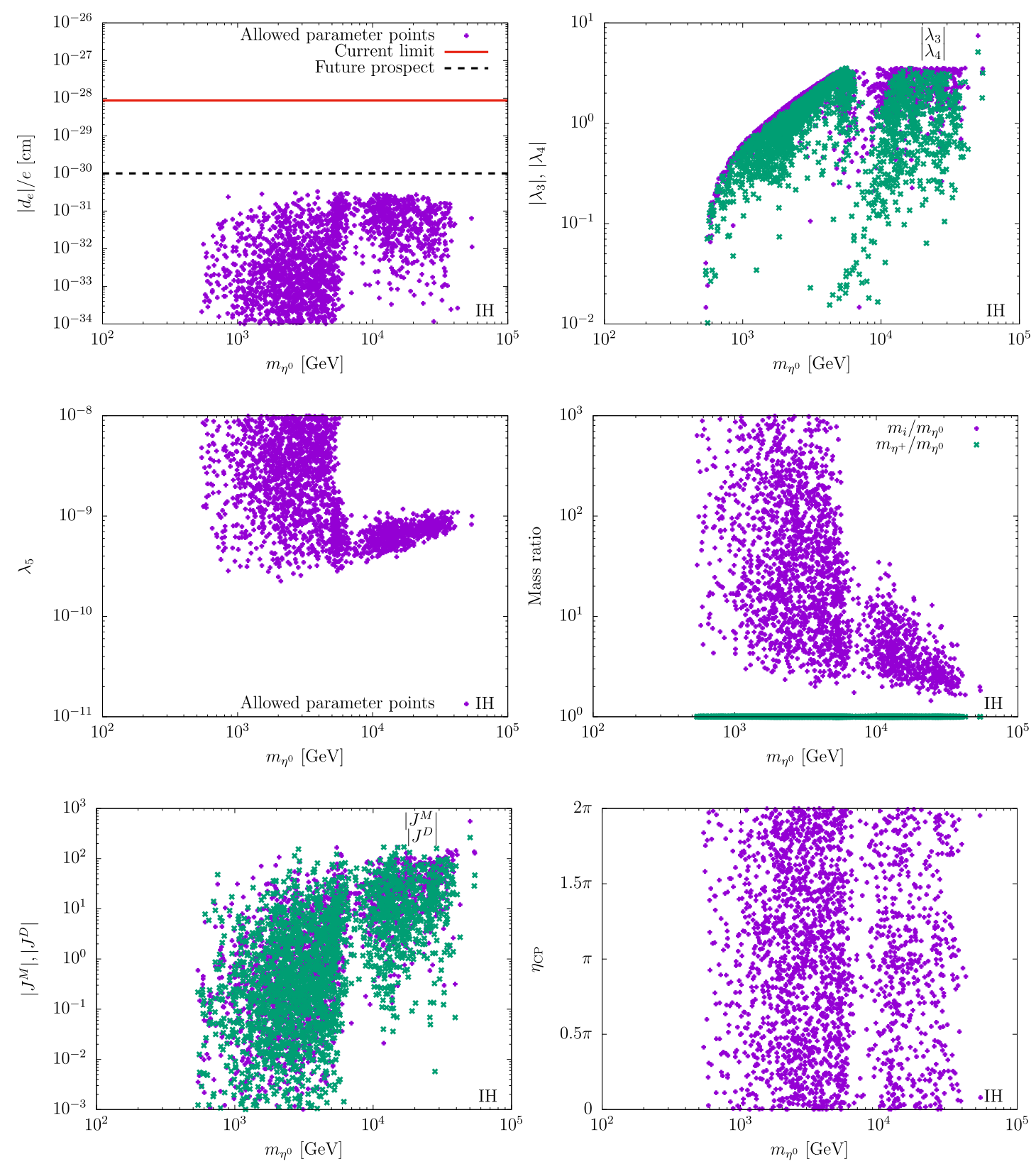

Figure 7. Numerical results for scalar dark matter in the case of inverted hierarchy for the light neutrino mass spectrum. Each point complies with all the constraints discussed in section 4 . 